\title{
Cationic lipid bioanalysis: understanding distribution of lipid nanoparticles for delivery of RNA therapeutics
}

\author{
Glen Hawthorne ${ }^{*, 1}$, Sarah Harbach ${ }^{1,2} \&$ Amanda Wilson ${ }^{1}$ \\ ${ }^{1}$ Translational Biomarkers and Bioanalysis, Drug Safety \& Metabolism, IMED Biotech Unit, AstraZeneca, Cambridge, UK \\ ${ }^{2}$ Department of Biology, University of York, York, UK \\ * Author for correspondence: glen.hawthorne@astrazeneca.com
}

First draft submitted: 11 December 2017; Accepted for publication: 18 December 2018; Published online: 1 March 2018

Keywords: cationic lipids $\bullet$ LC-MS/MS • lipid nanoparticles

\section{Emergence of lipid nanoparticles for delivery of RNA therapeutics}

RNA therapies significantly increase the possibility to selectively target previously undruggable targets in vivo compared with other modalities such as small molecules and antibodies. They include siRNAs, miRNAs, antisense oligonucleotides and mRNA, including CRISPR-Cas9, with the possibility to suppress gene expression, alter splicing, introduce new proteins, alter epigenetic regulation and even edit the genome [1].

The major barrier to RNA therapies reaching the clinic is delivery [2]. RNA is unstable and rapidly cleared from the blood by the liver and kidneys, making systemic administration and delivery of 'naked' or unshielded RNA difficult. In addition, lipid bilayers provide cells with a barrier to the uptake of large, charged molecules [1], therefore RNA does not easily cross cell membranes due to physiochemical properties such as anionic charge, high molecular weight and hydrophilicity [3].

Lipid nanoparticles (LNPs) provide RNA with protection from plasma endonuclease enzymes and enable RNA delivery to the proposed site of activity. A major component of LNPs is the cationic lipid, which serves to stabilize the LNP and provide some structural rigidity though association with the polyanionic ribose phosphate backbone of the RNA therapeutic.

In general, LNPs contain a cationic lipid plus other 'helper lipids', such as cholesterol, phosphatidylcholines and PEGylated lipids to stabilize and improve biocompatibility and transfection efficiency [4]. The cationic lipid is necessary for incorporation of negatively charged RNA and self-assembly of LNPs [5] whereby the RNA colocalizes with the lipid in the LNP structure. The cationic lipid is also responsible for interactions with cellular membranes, facilitating endocytosis and the subsequent release of RNA into the cytoplasm [4]. $N$-[1-(2,3-dioleyloxy)propyl]$N, N, N$-trimethylammonium chloride was the first cationic lipid reported to facilitate nucleic acid delivery [6]. There is interest in measuring the cationic lipid as a surrogate for the distribution of the LNP formulation.

\section{Lipid bioanalysis by LC-MS/MS}

Bioanalytical scientists interested in method development and validation of LC-MS/MS methods for smallmolecule drug quantitation, are familiar with the potentially interfering lipids in the form of phospholipids, sometimes manifesting their presence with ESI signal enhancements or suppression. Strategies exist for avoiding phospholipid-related matrix effects such as using solid phase or liquid-liquid extraction in combination with well thought chromatography $[7,8]$. When developing bioanalytical methods, phospholipids can be characterized into classes with easily monitored signature ions to map suitable regions or chromatographic windows in which to elute the small molecule analytes of interest. The positive ion scan of $\mathrm{m} / \mathrm{z} 184>184$ characterizes the most abundant glycerophosphatidyl choline polar head group [9]. Fortunately, the cationic lipids used within LNPs are modified from those most abundantly endogenous in plasma and tissue homogenates and these differences can be exploited through selective chromatography to avoid phospholipid elution regions of the chromatogram. The cationic lipids, for which the analytical strategies are described here, have $\log \mathrm{D}$ values in the order of 14-16 rendering them unsuitable for some phospholipid removal plates which remove phospholipids based on lipophilicity. Other 
phospholipid removal plates work by virtue of interaction of the phosphate polar head group and zirconia-coated beads, providing a potential to extract highly hydrophobic compounds within the LNPs selectively from endogenous lipophilic compounds. Regardless, the preference in our laboratory is to adopt simple protein precipitation methods in 96-well plates due to its ease of incorporation with established workflows.

\section{Chromatography options for LC-MS/MS bioanalysis of cationic lipids}

Analysis of lipids in the field of lipidomics is often achieved using readily available chromatography materials such as reversed-phase columns or silica-based columns for ion interaction chromatography. The hydrophobic chain, either saturated or unsaturated, provides ample area for van der Waal interactions with C18 and shorter alkyl chain phases to elicit long retention times. Generally in reversed-phase systems for lipid analysis the organic solvent choice includes methanol, ethanol, isopropyl alcohol (IPA) or mixtures of these with acetonitrile rather than just acetonitrile. While acetonitrile is a stronger solvent, with lipids methanol and alcohol mixtures provide a more predictable elution [8]. Indeed the high acetonitrile percentages needed to elute the lipids can sometimes effect an increase in retention using a hydrophilic interaction with polar groups on the HPLC column stationary phase. The ionic interaction of the cation and nonpolar retention mechanisms of the hydrophobic portion of a cationic lipid means that secondary interactions can be a nuisance. These interactions manifest as extreme peak tailing and in some cases a 'splurge' of analyte is seen rather than the desired discrete peak. In our laboratory, acceptable reversed-phase chromatography of cationic lipids is frequently obtained using a charged surface hybrid column [10].

An alternative to reversed-phase conditions for chromatographic analysis of cationic lipids is to use a polar stationary phase and a relatively nonpolar mobile phase consisting of $1: 1 \mathrm{v} / \mathrm{v}$ IPA:acetonitrile and water. Similar to hydrophilic interaction chromatography (HILIC) (note: these are not true HILIC conditions due to the presence of IPA) increasing the organic solvent mixture increases the retention of the cationic lipid. The advantage of using such solvent compositions over the reversed-phase conditions is the increased sensitivity presumably as a result of facile solvent droplet evaporation during ESI.

\section{Sample-handling procedures for cationic lipids}

Cationic lipids, like most lipids readily adsorb to plastics and are prone to oxidation. Storage instructions for them should be closely adhered to. There is a wealth of information available from supplier websites [11]. In general, stock materials are stored in a freezer under nitrogen, air is displaced above the material in the vial by blowing nitrogen across the top of the vial before quickly replacing the cap. In aqueous environments lipids will readily bind to polypropylene labware, to keep this to a minimum inclusion of an alcohol - methanol, ethanol, IPA in the storage solution is often employed. This precaution is a practical way to enable readily available plastic labware to be used rather than using glass.

Cationic lipids consist of a lipophilic tail attached to a polar head group, typically attached by an ester bond. The ester bond is often unstable in plasma and other biological matrices where esterase enzymes are present. In order to overcome this stability liability, samples are harvested into prechilled tubes handled on ice and plasma is prepared in a refrigerated centrifuge as soon as practical following collection. Acidification has been proven to be a simple and effective method of plasma sample stabilization in our laboratory.

Collection of tissue samples for determination of cationic lipids also requires special considerations. While tissues can be collected and immediately snap-frozen in liquid nitrogen to prevent degradation of lipids by cooling, the sample homogenization by its vigorous and mechanical nature is a heat generating process. Our laboratory uses a ceramic bead homogenizer for tissue preparation ahead of extraction and analysis. To control temperature in such a system, chilled homogenization buffer is added to the tube containing frozen tissue which is then returned to the freezer. Immediately prior to homogenization, ceramic beads are added to the tube so that the sample is homogenized while frozen and the tissue remains chilled.

\section{Conclusion}

So it is evident that there are bioanalytical strategies in place to be able to quantify cationic lipids in biological matrices. Using this quantitative information goes someway to help understand the behavior of the LNP within a test system. However, the overall analytical strategy for an mRNA therapeutic is somewhat complex and can require exposure evaluation of the mRNA and the translated protein. The utility of the cationic lipid measurement is further complicated by the unstable nature of LNPs, which precludes distinguishing between intact LNP and the released cationic lipid in circulation. Furthermore, with multiple end points required from a single administration 
of pharmaceutical, the sample volumes and handling need to be as minimal and simple as possible. These are the new challenges faced by bioanalysts working with new modality therapeutics and complex delivery systems.

\section{Open access}

This work is licensed under the Attribution-NonCommercial-NoDerivatives 4.0 Unported License. To view a copy of this license, visit http://creativecommons.org/licenses/by-nc-nd/4.0/

\section{Financial \& competing interests disclosure}

The authors have no relevant affiliations or financial involvement with any organization or entity with a financial interest in or financial conflict with the subject matter or materials discussed in the manuscript. This includes employment, consultancies, honoraria, stock ownership or options, expert testimony, grants or patents received or pending, or royalties.

No writing assistance was utilized in the production of this manuscript.

\section{References}

1. Dowdy SF. Overcoming cellular barriers for RNA therapeutics. Nat. Biotechnol. 35(3), 222-229 (2017).

2. Tam YY, Chen S, Cullis PR. Advances in lipid nanoparticles for siRNA delivery. Pharmaceutics 5(3), 498-507 (2013).

3. Semple SC, Akin A, Chen J et al. Rational design of cationic lipids for siRNA delivery. Nat. Biotechnol. 28(2), 172-176 (2010).

4. Cheng X, Lee RJ. The role of helper lipids in lipid nanoparticles (LNPs) designed for oligonucleotide delivery. Adv. Drug Deliv. Rev. 99, 129-137 (2016).

5. Jayaraman M, Ansell SM, Mui BL et al. Maximizing the potency of siRNA lipid nanoparticles for hepatic gene silencing in vivo. Angew. Chem. Int. Ed. Engl. 51(34), 8529-8533 (2012).

6. Felgner P, Gadek TR, Holm M et al. Lipofection: a highly efficient, lipid-mediated DNA-transfection procedure. Proc. Natl Acad. Sci. USA 84, 7413-7417 (1987).

7. Jemal M, Ouyang Z, Xia Y-Q. Systematic LC-MS/MS bioanalytical method development that incorporates plasma phospholipids risk avoidance, usage of incurred sample and well thought-out chromatography. Biomed. Chrom. 24(1), 2-19 (2010).

8. Silvester S, Smith L. Profiling phospholipid elution in reversed phase LC-MS/MS bioanalytical methods in order to avoid matrix effects. Bioanalysis 4(8), 879-895 (2012).

9. Little JL, Wempe MF, Buchanan CM. Liquid chromatography-mass spectrometry/mass spectrometry method development for drug metabolism studies: examining lipid matrix ionisation effects in plasma. J. Chromatogr. B Analyt. Technol. Biomed. Life Sci. 833(2), 219-230 (2006).

10. Isaac G, McDonald S, Astarita G. Lipid separation using UPLC with charged surface hybrid technology. Waters, MA, USA (2011). www.waters.com/webassets/cms/library/docs/720004107en.pdf

11. Avanti Polar Lipids, Inc. Storage and Handling of Lipids. AL, USA. https://avantilipids.com/tech-support/storage-handling-of-lipids/ 
